\section{Memory of water revisited}

SIR - Hirst et al.'s claim ${ }^{1}$ to have failed to replicate our findings ${ }^{2}$ (that high dilutions of anti-IgE can trigger basophil degranulation) is unfounded as they did not follow our methods ${ }^{2-7}$, and we question their statistical analysis.

We have noted fifteen discrepancies in methodology, of which three are cardinal errors: (1) the authors did not eliminate blood that was unreactive to ponderal anti-IgE antiserum, and which selfevidently would not react to the same highly diluted antiserum; (2) they did not test the high dilutions and controls (themselves inadequate, proper controls being diluted dummy antibody) on the same blood samples, an experimental sine qua non; and (3) they added a centrifugation step after incubation, thereby impairing accurate counting of activated basophils $^{2,5}$. These errors alone are enough to invalidate their whole attempt. The procedures involved in our basophil test are complex; it is a pity the authors did not consult us before embarking on it.

Nevertheless, we believe that their data, despite the methodological errors, do support our findings: the differences between active and control sessions are clearly evident in Figs 2 and 3 . The authors admit observing those differences, but they seek to explain them away by invoking some "unidentified part" of their procedure. They also try to disguise the differences by some inappropriate statistical analyses: Fig. 1, for example, spuriously combines the high dilution (but not ponderal anti-IgE) and control data, presumably to make the dilutions appear less active. The "comparison within treatment" analysis in Table 1 is weak; had they used the more powerful "comparison between treatments", the true significance of the data would have been revealed. Because of these lacunae, we requested sight of Hirst et al.'s raw data, but the authors refused to provide them.

The phenomenon we described ${ }^{2}$ has been confirmed by independent laboratories $^{8-10}$. Our full assessment of Hirst et al.'s paper is available on request. J. Benveniste

INSERMU 200,

32 rue des Carnets,

92140 Clamart, France

B. Ducot

A. Spira

INSERMU 292,

Hôpital de Bicêtre,

94275 Le Kremlin-Bicêtre Cedex, France

SIR - We agree with the conclusions of Hirst et al. ${ }^{1}$. In fact, nearly two years ago our research group published similar results ${ }^{11}$. We performed 24 blind experiments, of which 14 were independently evaluated by two investigators. No evi- dence was found for a difference between vortexed and non-vortexed dilutions of anti-IgE (tenfold serial dilutions in the range $10^{21}-10^{30}$ ) on the staining properties of human basophil leukocytes. In fact, it was concluded that extreme dilutions had no activity at all.

Some differences, however, were found between the investigators in the sense that researcher $X$ counted about 4 more basophils per sample than $Y$, this being about 5 per cent of the mean $(P<0.001$ Wilcoxon's test). Although these data are quite reasonable, they indicate that countings do differ between persons. The possibility could therefore not be excluded that there was a vortex-related effect in our experiments that had gone unnoticed. The observation by Benveniste that one person was better at performing positive experiments than others (personal communication) could support this line of thought. Unfortunately, Benveniste never supported an analysis of possible experimenter-dependence in experiments in which someone who normally achieves positive results counts a number of experiments simultaneously with other workers.

Meanwhile, one of the co-authors of ref. 2, Dr Sainte-Laudy, has adapted the procedure of basophil staining that he published nearly three years ago ${ }^{12}$. In that procedure, pre-incubation of basophils with ultra-low doses of histamine has been reported to modulate the degranulation triggered by anti-IgE. In this way passive sensitization is avoided. Also, instead of the rather painstaking evaluation of toluidin-stained basophils, another dye (alcian blue) is used which allows rapid and clear-cut basophil counts without time-consuming training. Following this protocol, double-blind experiments are currently being performed in five different laboratories in the United States, Ireland, Italy and France as well as in our laboratory in the Netherlands. These experiments are coordinated by Professor Roberfroid (Louvain, Belgium). The last word has not yet been spoken.

Fred A. C. Wiegant

Department of Molecular Cell Biology,

Utrecht University, Padualaan 8 ,

3584 CHUtrecht, The Netherlands

1. Hirst, S. J. etal. Nature 366. 525-527 (1993).

2. Davenas, E. et al. Nature 333.816-818 (1988)

3. Benveniste, J. et al. C. R. Acad. Sci. Paris 316, (série II), 461-466 (1991)

4. Beauvais, F. et al. J. Immunol. 144, 3881-3890 (1990).

5. Beauvais, F. et al. J. Allergyclin. Immun. 87, 10201028 (1991).

6. Beauvais. F. etal.J. Immun. 148, 149-154 (1992).

7. Beauvais, F. etal. Fundam. clin. Pharmac. 6.153-158 (1992).

8. Youcibier-Sismo, B. J. et al.Int. J. Immunother. 9. $169-180$ (1993)

9. Endler, P. C. etal. Vet Hum. Tox. 36, 56-59 (1994)

10. Bellen etal. Ag. Act. 38, 245-247 (1993).

11. Ovelgonne, J. H. et al. Experientia 48, 504-508 (1992)

12. Sainte-Laudy, J. in Uitra Low Doses (ed. Doutremepuich, C.) 139-143 (Taylor \& Francis, London, 1991)

\section{Proof positive}

SIR - I was gratified to note in the 12 May issue (Nature 369, 97; 1994) that John Maddox condemns the use of the phrase "evidence for" when the correct phrase should be "inconclusive evidence for". But I was astonished that on page 164 of the same issue Kevin Davies, the editor of Nature Genetics, uses the phrase ". . .but maybe these are just the exceptions that prove the rule". The context of the article forces one to assume that by these words he means that these exceptions demonstrate that the rule (that intra- and intergenic DNA sequences represent 'junk' DNA) is thus validated. 'Prove' derives from the Latin probare, meaning to try, approve or prove, and the first meaning is generally given as 'to test'. Examples are to prove gold, that is, to test its genuineness, or the noun 'proof' as applied to whisky where the test is whether it can be ignited or not. Hence an exception that proves the rule in fact tests the rule and if exceptions are found the rule is false.

\section{J. Edward Rall}

Bldg $10 \mathrm{Rm} 8 \mathrm{~N} 307$,

National Institute of Diabetes

and Digestive and Kidney Diseases,

Bethesda, Maryland 20892, USA

\section{Sophistry. . . ?}

SIR - Frank J. Tipler, in his review of Fire in the Equations by Kitty Ferguson ( $\mathrm{Na}$ ture 369,$198 ; 1994)$ quotes Hume as saying that books that contain neither abstract reasoning on quantity or number nor experimental reasoning on matter of fact and existence can contain only sophistry and illusion. In which category does Hume's statement belong?

Joseph Hertzlinger

16 Warren Lane,

Jericho, New York 11753, USA

\section{Missing person}

SIR-Your report that Sir Martin Rees is to be the new Astronomer Royal (Nature 370, 8; 1994) left a black (or, rather, white) hole where his photograph was expected. Are we to take this as evidence for the

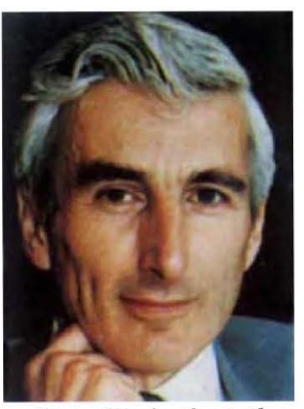
suspicion aroused by Star Trek that the task of the Astronomer Royal is to boldly see what no man has seen before?

\section{David Weltzman}

41 Hollybush Road, Cardiff CF2 6SY, UK

- The photograph appeared in some editions. NATURE · VOL 370 • 4 AUGUST 1994 